\title{
Michał Walicki
}

\section{MODALITIES AS INTERACTIONS BETWEEN THE CLASSICAL AND THE INTUITIONISTIC LOGICS}

\begin{abstract}
We give an equivalent formulation of topological algebras, interpreting S4, as boolean algebras equipped with intuitionistic negation. The intuitionistic substructure - Heyting algebra - of such an algebra can be then seen as an "epistemic subuniverse", and modalities arise from the interaction between the intuitionistic and classical negations or, we might perhaps say, between the epistemic and the ontological aspects: they are not relations between arbitrary alternatives but between intuitionistic substructures and one common world governed by the classical (propositional) logic. As an example of the generality of the obtained view, we apply it also to S5. We give a sound, complete and decidable sequent calculus, extending a classical system with the rules for handling the intuitionistic negation, in which one can prove all classical, intuitionistic and S4 valid sequents.
\end{abstract}

Keywords: topological algebras, boolean algebras, Heyting algebras, modal logics, intuitionistic logics.

\section{Introduction}

To make the paper self-contained, we begin in Section 2 by recalling the topological algebra semantics for propositional S4. Relations of topological algebras to Heyting algebras motivate an equivalent formulation of topological algebras as $\mathcal{I C}$-algebras, which are presented in Section 3. The only difference consists in replacing the closure/interior operation of topological 
algebras with intuitionistic negation. The categories of topological and $\mathcal{I C}$ algebras are isomorphic, but the reformulation makes explicit the relationships between the intuitionistic and classical propositional logics which, together, constitute S4. A technical advantage of this formulation, exemplified in Subsection 3.2, is that proofs of embeddings and dependencies between these three logics, performed traditionally at the metalevel and typically by the analysis of the respective proof systems, become internalized in the common language of $\mathcal{I C}$-algebras. Section 4 shows how the new formulation adapts to extensions of S4, exemplified by S5. In Section 5, we augment the sequent calculus for classical logic with two rules for handling the intuitionistic negation, and prove its soundness and completeness with respect to the class of all $\mathcal{I C}$-algebras. A simple argument for the decidability of the calculus is given. The proofs and technical results of the paper are relatively straightforward (details omitted here can be found in [10]) and a reader familiar with the algebraic semantics of modalities can consult directly definition 2 in Section 3 and the calculus in section 5. However, the emerging observation that modalities arise as combinations of classical and intuitionistic negation is novel and the concluding Section 6 lists some philosophical implications of this fact.

\section{Background}

Definition 1. A toplogical algebra $\mathbf{T}=\langle T ; \cap, \cup,-, \mathbf{c}\rangle$ is a boolean algebra $\langle T ; \cap, \cup,-\rangle$ with a closure operator $\mathbf{c}: T \rightarrow T$ (or, equivalently, interior $\mathbf{i}(x)=-\mathbf{c}(-x))$, satisfying the equations:
c1. $x \subseteq \mathbf{c}(x)$
i1. $x \supseteq \mathbf{i}(x)$
c2. $\mathbf{c}(\mathbf{c}(x))=\mathbf{c}(x)$
i2. $\mathbf{i}(\mathbf{i}(x))=\mathbf{i}(x)$
c3. $\mathbf{c}(x \cup y)=\mathbf{c}(x) \cup \mathbf{c}(y)$
i3. $\mathbf{i}(x \cap y)=\mathbf{i}(x) \cap \mathbf{i}(y)$
c4. $\mathbf{c}(\mathbf{0})=\mathbf{0}$
i4. $\mathbf{i}(\mathbf{1})=1$

An element $x \in T$ is open/closed iff $x=\mathbf{i}(x) / x=\mathbf{c}(x)$.

Closure, resp. interior, interprets the modality $\diamond$, resp. $\square$, and S4 logic is sound and complete with respect to the class of all topological algebras.

Such an algebra contains a Heyting algebra of open elements, namely, $I(\mathbf{T})=\langle O ; \cap, \cup, \hookrightarrow, \mathbf{0}\rangle$, where

I1. $O=\{x \in T \mid x=\mathbf{i}(x)\}$

I2. $\cup, \cap$ and $\mathbf{0}$ are inherited from $\mathbf{T}$

I3. $\forall x, y \in O: x \hookrightarrow y=\mathbf{i}(-x \cup y)$ 
One verifies easily that $I$ can be extended to a functor from the category $\mathcal{T A}$ of topological algebras (with homomorpisms respecting $\cap, \cup,-$, c as morphisms) to the category $\mathcal{H} \mathcal{A}$ of Heyting algebras (with homomorphisms respecting $\cap, \cup, \hookrightarrow, \mathbf{0}$ as morphisms).

On the other hand, any Heyting algebra, $\mathbf{H}$, can be extended in a canonical way to a topological algebra, $\mathbf{T}=C(\mathbf{H})$.

Theorem 2.1. There is a functor $C: \mathcal{H} \mathcal{A} \rightarrow \mathcal{T A}$, such that $C ; I=I D_{\mathcal{H} \mathcal{A}}$.

Proof. That every Heyting algebra can be obtained as algebra of open elements of a toplogical algebra is the theorem 1.15 from [6] (formulated dually in terms of closed elements), or IV.3.1 from [7]. We only sketch the main aspects of the construction.

Given $\mathbf{H}=\langle H ; \sqcap, \sqcup, \hookrightarrow, \mathbf{0}\rangle$, we consider it first as a bounded distributive lattice $\langle H, \sqcap, \sqcup, \mathbf{0}, \mathbf{1}\rangle$. By [4], $\mathbf{H}$ can be extended uniquely to a boolean algebra $\mathbf{T}=\langle T ; \cap, \cup,-\rangle$ where

1. $\mathbf{H}$ is a sublattice of $\mathbf{T}$ (i.e., $\forall x, y \in H: x \cup y=x \sqcup y$ and $x \cap y=x \sqcap y$ )

2. every element $b \in T$ is of the form $\bigcap_{1}^{n}-a_{i} \cup b_{i}$ for some finite set of $a_{i}, b_{i} \in H$.

Using the fact that $a \hookrightarrow b \subseteq-a \cup b$, one shows that the choice of representatives in 2 is inessential for the definition of the interior/closure operator. $\forall a_{i}, b_{i}, a_{j}, b_{j} \in H$ :

$$
\bigcap_{1}^{n}-a_{i} \cup b_{i}=\bigcap_{1}^{m}-a_{j} \cup b_{j} \Rightarrow \bigcap_{1}^{n} a_{i} \hookrightarrow b_{i}=\bigcap_{1}^{m} a_{j} \hookrightarrow b_{j}
$$

So, for every element of $T$ (of the form 2), interior can be defined as:

$$
\mathbf{i}\left(\bigcap_{1}^{n}-a_{i} \cup b_{i}\right)=\bigcap_{1}^{n} a_{i} \hookrightarrow b_{i}
$$

Letting $C(\mathbf{H})=\mathbf{T}$ gives $I(C(\mathbf{H}))=\mathbf{H}$.

Verification of functoriality is based on the above representation but its technicalities do not contribute to the present paper. Details can be found in [10].

The canonicity of the extension mentioned above refers to the fact that the functors are adjoint, $C \dashv I,[10]$. For the following considerations, the crucial observation is that in any $\mathcal{T} \mathcal{A}$-algebra one can define, according to $\mathbf{I 3}$, the operation corresponding to the intuitionistic negation by $\div x=\mathbf{i}(-x)$. 
On the other hand, according to (2.3), this equation holds in the topological extension $C(\mathbf{H})$ of a Heyting algebra $\mathbf{H}$, i.e., the intuitionistic negation "survives" this extension, albeit it gets "hidden within" $\mathbf{i}$.

\section{3. $\mathcal{I C}$-algebras}

For every Heyting algebra $\mathbf{H}$ and $x \in H$, we obtain in the extension $\mathbf{T}=$ $C(\mathbf{H})$ :

$$
\mathbf{c}(x)=-\mathbf{i}(-x)=-\mathbf{i}(-x \cup \mathbf{0}) \stackrel{(2.3)}{=}-(x \hookrightarrow \mathbf{0})=-\div x,
$$

This fact that the closure/interior operation of any topological algebra $\mathbf{T}$ contains an aspect of intuitionistic negation is what makes the straightforward reduction of such algebras to Heyting algebras possible when defininig the object part of the functor $I, \mathbf{I} 1-\mathbf{I} 3$. (3.1) gives also the dual fact:

$$
\mathbf{i}(x)=\div-x
$$

The equations (3.1), (3.2) might look suspicious since $\div$ is defined only over H. But as observed at the end of the previous section, $\div$ (as well as the relative pseudo-complement $\hookrightarrow$ ) can be introduced in any $\mathcal{T} \mathcal{A}$-algebra:

$$
\text { i) } \div x=\mathbf{i}(-x) \quad \text { ii) } x \hookrightarrow y=\mathbf{i}(-x \cup y)=\div-(-x \cup y) \text {. }
$$

This suggests the possibility of combining in one structure the classical and intuitionistic elements according to the following definition.

Definition 2. An $\mathcal{I C}$-algebra ("intuitionistic-classical") is a tuple $\langle C ; \cup, \cap$, $-, \div\rangle$ where $\langle C ; \cup, \cap,-\rangle$ is a boolean algebra, and a unary operation $\div$ (intuitionistic negation) satisfies the following axioms:

$$
\begin{array}{ll}
\text { s1. } \div x \subseteq-x & \text { s3. } \div(x \cup y)=\div x \cap \div y \\
\text { s2. } \div x=\div-\div x & \text { s4. } \div \mathbf{0}=\mathbf{1}
\end{array}
$$

The formulations of $\mathbf{s 1 ,} \mathbf{s} \mathbf{2}$ are equivalent to those in $\mathbf{i} \mathbf{1}$ and $\mathbf{i} \mathbf{2}$ (see $\mathbf{1 1}$, $\mathbf{1 2}$ below). s4 is equivalent to $\mathbf{i} 4$, and $\mathbf{s} 3$ to $\mathbf{i} 3$. Hence, every $\mathcal{T} \mathcal{A}$-algebra can be converted into such an $\mathcal{I C}$-algebra using (3.3).i), while an $\mathcal{I C}$-algebra can be converted into a $\mathcal{T} \mathcal{A}$-algebra using (3.2). For instance, every topological space, being a $\mathcal{T} \mathcal{A}$-algebra, can be now seen also as an $\mathcal{I C}$-algebra, where the operation $\div x$ gives, according to (3.3), the interior of the complement of $x$.

Given the mutual interdefinability of $\div$ and $\mathbf{i}$ in the presence of the boolean operations, one verifies easily that also $\mathcal{T A}$ and $\mathcal{I C}$ homomorphisms 
coincide: the categories of $\mathcal{I C}$ and of $\mathcal{T A}$ algebras are isomorphic. We have thus not changed anything in the semantics, but merely given a different language for describing it.

\subsection{Some tautologies}

The change of the language gives, however, a different perspective with which we will be concerned in the rest of the paper. In particular, it brings forth the interaction between the classical and intuitionistic negations hidden under $\square$ and $\diamond$. The following tautologies provide some examples. On the right we give more familiar (and sometimes more specific) formulations in one of the sublanguages.

11. $\mathbf{s 1} \Leftrightarrow$ i1, i.e., $\div x \subseteq-x \Longleftrightarrow \div-x \subseteq x \ldots \ldots \ldots \ldots \ldots . \square x \rightarrow x$

12. s2 $\Leftrightarrow$ i2, i.e., $\div x=\div-\div x \Longleftrightarrow \div-x=\div-\div-x \quad . \quad \square x \leftrightarrow \square \square x$

13. $\div 1=0$

14. $x \subseteq y \Rightarrow \div x \supseteq \div y \ldots \ldots \ldots \ldots \ldots \ldots \ldots . \ldots . \ldots . \ldots(x \hookrightarrow y) \hookrightarrow(\div y \hookrightarrow \div x)$

15. $\div-(\div x \cup \div y)=\div x \cup \div y \ldots \ldots \ldots \ldots . \mathbf{i}(\mathbf{i}(x) \cup \mathbf{i}(y))=\mathbf{i}(x) \cup \mathbf{i}(y)$

16. $\div x=x \hookrightarrow \mathbf{0}$, using (3.3).ii) as the definition of $\hookrightarrow$

17. $x \cap \div x=0$

18. $a \cap x \subseteq b \Leftarrow a \subseteq x \hookrightarrow b$

19. $a \cap x \subseteq b \Rightarrow a \subseteq x \hookrightarrow b$, when (*) $a=\div a^{\prime}$

110. $x \subseteq \div \div x$, when $\left({ }^{*}\right) x=\div x^{\prime} \ldots \ldots \ldots \ldots \ldots \ldots \ldots \ldots \ldots \ldots+\div$.

111. $\div-x \subseteq \div \div x \ldots \ldots \ldots \ldots \ldots \ldots \ldots \ldots \ldots \ldots \ldots, \square x \rightarrow \square \diamond x$

112. $\div x \cup \div y \subseteq \div(x \cap y) \ldots \ldots \ldots \ldots \ldots \ldots \ldots \ldots \ldots \ldots \ldots \ldots \ldots \ldots+(x \wedge y)$

113. $\div \div x=\div \div \div \div \ldots \ldots \ldots \ldots \ldots \ldots \ldots \ldots \ldots \ldots \ldots \ldots+x \leftrightarrow \div \div \div$

114. $\div x=1 \Rightarrow x=0$

115. $\div x=0 \nRightarrow x=1$

\subsection{Relating tautologies}

The above lemmata give only a few examples of a vast variety of tautologies. We can easily conclude that the following hold in $\mathcal{I C}$-algebras (the respective restriction of the language is given to the right):

$\beta$ ) any classical (boolean) tautology $\quad \beta::=x|\beta \cap \beta| \beta \cup \beta|-\beta| \mathbf{0}$

$\mu$ ) any topological/S4 tautology $\quad \mu::=x|\mu \cap \mu| \mu \cup \mu|-\mu| \div-\mu \mid \mathbf{0}$

$\iota)$ any intuitionistic tautology $\iota::=\div-x|\iota \cap \iota| \iota \cup \iota|\div-(-\iota \cup \iota)| \mathbf{0}$ 
We let $\gamma$ denote the grammar of the whole language. (In $\beta$ and $\mu$, we added $\mathbf{0}$ merely to ease comparison.) The restriction on the variables in $\iota$ ensures that all intuitionistic formulae address only the intuitionistic/open elements of the algebras. (Equivalently, we might only require $\div x$.) For instance, 113 would be formulated intuitionistically with one $\div$ less. In our case, it acquires this additional $\div$ as the intuitionistic tautology, $\div x=\div \div \div x$, holds for the open, but not necessarily for other elements. Similarly, the intuitionistic tautology $\mathbf{1 1 0}$ may fail when $x$ is not open. On the other hand, some intuitionistic tautologies survive unchanged and can be applied to all elements, not only the open ones, e.g., 18, 112. Note also that, although $L(\mu) \neq L(\gamma)$, so for every $\phi \in L(\gamma)$ there is an equivalent formula $\psi \Leftrightarrow \phi$ with $\psi \in L(\mu)$, because $\div$ is definable in $L(\mu)$ as $\div x=\div-(-x)$.

Validity of all (instances of) classical tautologies follows since $\mathcal{I C}$-algebras are boolean algebras, and validity of all topological tautologies since they are also topological algebras. Validity of all intuitionistic tautologies follows since, by the restriction on the variables which must be preceded by $\div-$, they address only open elements of an $\mathcal{I C}$-algebra, that is, only and all elements of its substructure which is Heyting algebra. By theorem 2.1, every Heyting algebra is a substructure of some $\mathcal{I C}$-algebra.

In addition, we have tautologies, e.g., $\mathbf{s 1}$ or $\mathbf{s} \mathbf{2}$, which do not belong to any of these sublanguages but, so to speak, express "connections" between them. They allow us to formulate and verify some of the classical results relating the different logics in the internal language of $\mathcal{I C}$.

3.2.1. An example of such a result is McKinsey-Tarski embedding of IL into S4:

$$
\begin{aligned}
\mathrm{IL} & \mapsto \mathrm{S} 4 \\
\hline a \in X: \operatorname{tr}(a) & =\square a \\
\operatorname{tr}\left(\phi_{1} \wedge \phi_{2}\right) & =\operatorname{tr}\left(\phi_{1}\right) \wedge \operatorname{tr}\left(\phi_{2}\right) \\
\operatorname{tr}\left(\phi_{1} \vee \phi_{2}\right) & =\operatorname{tr}\left(\phi_{1}\right) \vee \operatorname{tr}\left(\phi_{2}\right) \\
\operatorname{tr}\left(\phi_{1} \rightarrow \phi_{2}\right) & =\square\left(\operatorname{tr}\left(\phi_{1}\right) \rightarrow \operatorname{tr}\left(\phi_{2}\right)\right)
\end{aligned}
$$

In the present formulation, this embedding of syntax becomes simply an inclusion $\operatorname{tr}\left(\_\right): L(\iota) \subset L(\mu)$. The statement $\Gamma \models \phi \Longleftrightarrow \operatorname{tr}(\Gamma) \models \operatorname{tr}(\phi)$ becomes now a consequence of the fact that functor $I$ is surjective on the objects, i.e., that every Heyting algebra can be obtained as an algebra of opens of some topological algebra.

3.2.2. The intuitionistic logic emerges as a syntactic subset of $\mathcal{I C}$-logic. Specificity of its connectives, in particular, disjunction, can be thus seen as 
a consequence of restricting the domain of interpretation, which is reflected in the basic case of its grammar: it addresses only the open elements. (One checks easily (using $\mathbf{s} \mathbf{2}, \mathbf{s} 3$ and $\mathbf{1 5}$ ) that elements interpreting $L(\iota)$-formulae are open.)

As an example illustrating that we obtain "intuitionistic" connectives by restricting attention to the "intuitionistic" elements, we show the disjunction property:

LEMma 3.4. The following implications hold ( $x$ may be a sequence of variables):

1. If $\mathcal{I C} \models-\div \phi_{1}(x) \cap-\div \phi_{2}(x)=\mathbf{0}$, then $\mathcal{I C} \models \phi_{1}(x)=\mathbf{0}$ or $\mathcal{I C} \models$ $\phi_{2}(x)=\mathbf{0}$.

2. If $\mathcal{I C} \models \div \phi_{1}(x) \cup \div \phi_{2}(x)=\mathbf{1}$, then $\mathcal{I C} \models \div \phi_{1}(x)=\mathbf{1}$ or $\mathcal{I C} \models \div \phi_{2}(x)=$ 1.

Proof. 1 is the theorem 4.12 from [5] $(-\div x=\mathbf{c}(x))$. It holds here because each $\mathcal{I C}$-algebra can be seen as a toplogical algebra (used in that theorem) and vice versa.

The disjunction property 2 follows from 1 . The assumption is equivalent to $\mathcal{I C} \models\left(-\div \phi_{1}(x)\right) \cap\left(-\div \phi_{2}(x)\right)=\mathbf{0}$. Then either $\mathcal{I C} \models \phi_{1}(x)=\mathbf{0}$ or $\mathcal{I C} \models \phi_{2}(x)=\mathbf{0}$, by 1 . In either case, $\div \phi_{i}(x)=\mathbf{1}$ by $\mathbf{s} \mathbf{4}$.

3.2.3. Since $\div$ is the "switch" which brings an element over into the "intuitionistic subuniverse", some classical results, like those involving negative translations, obtain an internal expression. For instance, $a=\mathbf{1} \stackrel{13}{\Longrightarrow} \div a=$ $\mathbf{0} \stackrel{\mathrm{s} 4}{\Longrightarrow} \div a=1$, gives the general statement:

$$
\mathcal{I C} \models \phi(x)=\mathbf{1} \Longrightarrow \mathcal{I C} \models \div \div \phi(x)=\mathbf{1} .
$$

In a sense, this is stronger than the classical result, since $\phi$ can now contain both classical and intuitionistic connectives. But for this reason it gives a weaker relation between the respective logics.

A series of classical metatheorems arise from $\mathcal{I C}$-tautologies, and we give only one example. As a consequence of (3.5), when $\phi$ is an intuitionistic tautology, so is $\div \div \phi$. But our $\div \div \phi$ may involve non-intuitionistic expressions. One direction of Glivenko's theorem will have the following form. ${ }^{1}$ When

\footnotetext{
${ }^{1}$ We do not address the opposite implication which is a trivial consequence of the completeness results for IL and CL, and the observation that IL-provability is contained in CL-provability.
} 
$\phi(x) \in L(\beta)$ then:

$$
\mathcal{I C} \models \phi(x)=\mathbf{1} \Longrightarrow \mathcal{I C} \models \div \div \phi^{\prime}(\div-x)=\mathbf{1}
$$

where $\phi^{\prime}$ is $\phi$ with all $-/ \rightarrow$ replaced by $\div / \hookrightarrow$.

Assume first $\phi$ to be in CNF, i.e., $\bigcap_{i}\left(\bigcup_{j} \bar{x}_{i j}\right)$ where each $\bar{x}$ is $x$ or $-x$. We conduct the proof for an arbitrary $\mathcal{I C}$-algebra $\mathbf{T} \models \phi=\mathbf{1}$ :

$$
\begin{aligned}
& \bigcap_{i}\left(\bigcup_{j} \bar{x}_{i j}\right)=\mathbf{1} \Longleftrightarrow \bigcup_{j} \bar{x}_{i j}=\mathbf{1} \\
& \Longleftrightarrow \quad \div-\bigcup_{j} \bar{x}_{i j}=1 \\
& =\quad \div-\left(\bigcup_{i j n}-x_{i j n} \cup \bigcup_{i j p} x_{i j p}\right)=\mathbf{1} \\
& \Longleftrightarrow \quad \div\left(\bigcap_{i j n} x_{i j n} \cap \bigcap_{i j p}-x_{i j p}\right)=\mathbf{1} \\
& \stackrel{\text { subst) }}{\Longrightarrow} \div\left(\bigcap_{i j n} \div \div x_{i j n} \cap \bigcap_{i j p}-\div \div x_{i j p}\right)=1 \quad x \mapsto \div \div x \\
& \stackrel{113}{\Longleftrightarrow} \div\left(\bigcap_{i j n} \div \div \div \div x_{i j n} \cap \bigcap_{i j p}-\div \div x_{i j p}\right)=1 \\
& \stackrel{\text { s1,14 }}{\Longrightarrow} \div\left(\bigcap_{i j n} \div \div \div \div x_{i j n} \cap \bigcap_{i j p} \div x_{i j p}\right)=1 \quad x \subseteq-\div x \\
& \stackrel{\text { s3 }}{\Longleftrightarrow} \div \div\left(\bigcup_{i j n} \div \div \div x_{i j n} \cup \bigcup_{i j p} x_{i j p}\right)=1 \\
& \stackrel{\text { subst) }}{\Longrightarrow} \div \div\left(\bigcup_{i j n} \div \div \div \div-x_{i j n} \cup \bigcup_{i j p} \div-x_{i j p}\right)=1 \quad x \mapsto \div-x \\
& \stackrel{113}{\Longleftrightarrow} \div \div\left(\bigcup_{i j n} \div \div-x_{i j n} \cup \bigcup_{i j p} \div-x_{i j p}\right)=1 \\
& =\quad \div \div\left(\bigcup_{i j n} \div x_{i j m}^{\prime} \cup \bigcup_{i j p} x_{i j p}^{\prime}\right)=1 \\
& =\quad \div \div \bigcup_{i j} \bar{x}_{i j}^{\prime}=\mathbf{1} \\
& \Longleftrightarrow \bigcap_{i} \div \div \bigcup_{j} \bar{x}_{i j}^{\prime}=\mathbf{1} \\
& \stackrel{\text { s3 }}{\Longleftrightarrow} \div \bigcup_{i} \div \bigcup_{j} \bar{x}_{i j}^{\prime}=1 \\
& \stackrel{\mathrm{l12}}{\Longrightarrow} \div \div \bigcap_{i} \bigcup_{j} \bar{x}_{i j}^{\prime}=1 \\
& x^{\prime}=\div-x \\
& \text { for all } i
\end{aligned}
$$

(All lines between the first and the last one marked "for all $i$ " carry this condition.) The resulting $\bar{x}_{i j}^{\prime}$ have the form $\div-x_{i j}$ and those which were preceded by - are now preceded by $\div$ instead (line $-5 /-4$ ).

To complete the proof for arbitrary tautologies, not only in CNF, we only observe that any $\div \div \phi(x) \in L(\iota)$ is (intuitionistically) equivalent to $\div \div \phi^{\prime}(x)$ where $\phi^{\prime}$ is obtained from $\phi$ by classical transformations (e.g., $\div \div\left(\div \psi_{1}(x) \cup \div \psi_{2}(x)\right)=\div \div \div\left(\psi_{1}(x) \cap \psi_{2}(x)\right), \div \div\left(\div \psi_{1}(x) \cup \psi_{2}(x)\right)=$ $\div \div\left(\psi_{1}(x) \hookrightarrow \psi_{2}(x)\right)$, etc. Hence, if our classical tautology is not, initially, in $\mathrm{CNF}$, we transform it into CNF, apply the above result, and then transform the final intuitionistic formula under $\div \div$ into the corresponding intuitionistic form using these equivalences.

\section{4. $\mathcal{I C}$-models for $\mathrm{S} 5$}

The development in Section 3 is not limited to S4. What is specific about S4 is only that it contains the intuitionistic logic in an unmodified form. 
Further extensions will, typically, affect this aspect and we illustrate it by an extension to S5.

To the $\mathcal{I C}$-axioms s1-s4, we add the S5-axiom:

s5. $-\div x \subseteq \div \div x$

which is just $-\div x \subseteq \div--\div x$, i.e., $\diamond x \rightarrow \square \diamond x$. Combined with axiom s1, this entails $-\div x=\div \div x$. That is, in $\mathcal{I C}$-algebras for $\mathrm{S} 5$, the negation of open elements equals the interior of their negation, i.e., the complement of an open is open.

An equivalent definition of S5-algebras, e.g., [2], requires that complement of every closed element is closed, i.e.,

(*) $\forall x \exists y:-(-\div x)=-\div y$.

(s5 $\Rightarrow\left({ }^{*}\right)$ follows since $-\div x=\div \div x \Rightarrow-(-\div x)=-\div(\div x)$, so we can take $y=\div x$. For the opposite implication, let $x$ be arbitrary, then $-\div x$ is closed, and so $-(-\div x) \stackrel{(*)}{=}-\div y$, i.e., $\div x=-\div y$. But then $-\div(\div x)=-\div-\div y=-\div y=\div x \Rightarrow \div \div x=-\div x$. $)$ As is well known, a topological algebra is an S5-algebra iff the topology is almost discrete (open $=$ closed).

In such algebras, we obtain, for instance:

s5-11. $-\div \div-x=\div-x$ i.e., $\diamond \square x \leftrightarrow \square x$

s5-12. $\div \div \div x=-\div \div x=-\div x=\div x$

The essential difference between the $\mathcal{I C}$-algebras for $\mathrm{S} 4$ and for $\mathrm{S} 5$ is that the former contain genuine Heyting substructures. In the latter, where complement of an open is open, every open element $\div x$ is regular, 2 , which implies that the Heyting substructure is actually boolean. This known fact can be now seen as the crucial collapse enforced by S5: its modalities, still present, express no longer a relation between a classical world and its intuitionistic substructure, but between one classical world and its substructure which is itself classical.

\section{Reasoning}

Since $\mathcal{I C}$-algebras are boolean algebras with the additional operation of $\div$, the reasoning system is obtained by augmenting a sequent system for classical logic with the two rules for handling this connective. The rules given in Table 1 form a sound and complete reasoning system, LIC, for $\mathcal{I C}$-algebras. 
Having established some auxiliary results in 5.1, we prove completeness in 5.2. In 5.3 we give a decidability argument.

$$
\begin{array}{rll}
A x: & p \vdash p \text { for atomic } p & \\
& L \vdash & \vdash R \\
- & \frac{\Gamma \vdash \Delta, A}{\Gamma,-A \vdash \Delta} & \frac{\Gamma, A \vdash \Delta}{\Gamma \vdash \Delta,-A} \\
\rightarrow \quad & \frac{\Gamma \vdash \Delta, A ; \Gamma, B \vdash \Delta}{\Gamma, A \rightarrow B \vdash \Delta} & \frac{\Gamma, A \vdash \Delta, B}{\Gamma \vdash \Delta, A \rightarrow B} \\
\vee & \frac{\Gamma, A \vdash \Delta ; \Gamma, B \vdash \Delta}{\Gamma, A \vee B \vdash \Delta} & \frac{\Gamma \vdash \Delta, A, B}{\Gamma \vdash \Delta, A \vee B} \\
\wedge & \frac{A, B, \Gamma \vdash \Delta}{A \wedge B, \Gamma \vdash \Delta} & \frac{\Gamma \vdash A, \Delta ; \Gamma \vdash B, \Delta}{\Gamma \vdash A \wedge B, \Delta} \\
\therefore & \frac{\Gamma, \div A \vdash A, \Delta}{\Gamma, \div A \vdash \Delta} & \frac{\div \Gamma, A \vdash}{\div \Gamma \div A} \\
(W) & \frac{\Gamma \vdash \Delta}{A, \Gamma \vdash \Delta} & \frac{\Gamma \vdash \Delta}{\Gamma \vdash \Delta, B} \\
& &
\end{array}
$$

Table 1. The calculus LIC.

In the rule $(R \div), \div \Gamma$ denotes a sequence of formulae each starting with $\div$.

Each side of a sequent is a set of formulae. A sequent $\Gamma \vdash \Delta$ is valid iff for every $\mathcal{I C}$-algebra $M$ and every valuation of the variables occurring in the sequent, $v: X \rightarrow M, \cap v(\Gamma) \subseteq \bigcup v(\Delta)$, where valuations are extended to (sets of) formulae in the obvious way. ${ }^{2}$

Lemma 5.1. All rules are sound, i.e., for every rule $\frac{\Gamma_{i} \vdash \Delta_{i}}{\Gamma \vdash \Delta}$, for every $\mathcal{I C}$ algebra $M$ and every valuation $v$, if $\bigcap v\left(\Gamma_{i}\right) \subseteq \bigcup v\left(\Delta_{i}\right)$ then $\bigcap v(\Gamma) \subseteq \bigcup v(\Delta)$. The opposite implication [invertibility] holds for all rules except $(W)$.

\footnotetext{
${ }^{2}$ One might expect this definition to require: $\left(^{*}\right) \bigcap v(\Gamma)=\mathbf{1} \Rightarrow \bigcup v(\Delta)=\mathbf{1}$. This, however, would give, for instance, validity of $x \vdash \square x$ or $\square x, x \rightarrow y \vdash \square y$, which aren't sound for S4. (Note that $\square x, x \hookrightarrow y \vdash \square y$ does hold - it is actually the $K$ axiom: $\square x, \square(x \rightarrow y) \vdash \square y$.) Our definition implies $\left(^{*}\right)$, so any valid sequent/tautology is also valid according to $(*)$. Finally, it squares well with the empty rhs of $\Gamma \vdash \varnothing$ which becomes $\bigcap \Gamma=\mathbf{0}$ rather than $\bigcap \Gamma \neq \mathbf{1}$ : the rule $(R \div)$ is both sound and invertible. All rules are sound also with respect to $(*)$, if only we interepret $\Gamma \vdash \varnothing$ in $(R \div)$ as $\bigcap v(\Gamma)=\mathbf{0}$.
} 
Proof. The proof for all classical rules is standard and applies since $M$ is, in particular, a boolean algebra. We only show the claim for $(L \div)$ and $(R \div)$.

$(R \div) \cap \div \Gamma \cap A \subseteq \mathbf{0} \Longleftrightarrow \bigcap \div \Gamma \subseteq-A \Longleftrightarrow \bigcap \div \Gamma \subseteq \div A-$ the last equivalence holds since $\bigcap \div \Gamma$ is open. ${ }^{3}$

$(L \div) \Gamma \cap \div A \subseteq A \cup \Delta \Longleftrightarrow \Gamma \cap \div A \cap-A \subseteq \Delta \Longleftrightarrow \Gamma \cap \div A \subseteq \Delta-$ the last equivalence holds since $\div A \subseteq-A$.

Example 5.2. The formula $A \vee \div A$ is not provable, since the proof cannot proceed past the step $\vdash A, \div A$. Below, we give a proof of $\div \div(A \vee \div A)$ :

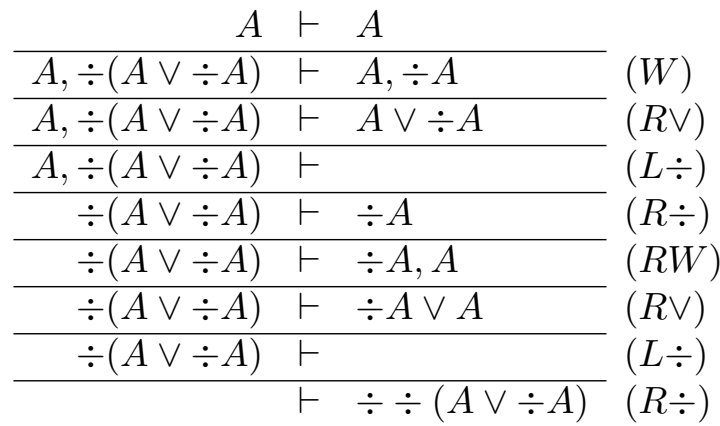

The "intuitionistic" tautology $A \hookrightarrow \div \div A$ is not provable. As observed at the beginning of Section 3.2, this is due to the fact that $A$ need not be interepreted as an open element of an $\mathcal{I C}$-algebra. Imposing such a requirement, gives the provable formula $\div A \hookrightarrow \div \div \div A$.

LEMma 5.3. The following rules are admissible:

1. (cut) $\frac{\Gamma \vdash \Delta, A ; \quad \Gamma^{\prime}, A \vdash \Delta^{\prime}}{\Gamma, \Gamma^{\prime} \vdash \Delta, \Delta^{\prime}}$

2. $\left(\mathrm{L}^{\prime} \div\right) \frac{\Gamma \vdash A, \Delta}{\Gamma, \div A \vdash \Delta}$

3. (T) $\frac{\Gamma, A \vdash \Delta}{\Gamma, \square A \vdash \Delta}$

$\left(\mathrm{S}^{\prime}\right) \frac{\div \Gamma \vdash A}{\div \Gamma \vdash \square A}$

(S4) $\frac{\square \Gamma \vdash A}{\square \Gamma \vdash \square A}$

\footnotetext{
${ }^{3}$ We do not have the distinction between the bound and free variables, and hence, between the open and closed formulae in the usual sense. Therefore, it should not be confusing if we call a formula "open"/"closed" when it denotes an open/closed element for all possible valuations.
} 
Proof. 1. The proof by induction (i) on the complexity of the cut formula and, secondarily, (ii) on the sum of the heights of the derivations of the premisses is standard - new cases for $\div$ present no serious difficulties. Details are given in [10].

2. $\left(\mathrm{L}^{\prime} \div\right) \quad \Gamma \vdash A, \Delta$

$$
\begin{array}{ll}
\Gamma, \div A \vdash A, \Delta & (L W) \\
\Gamma, \div A \vdash \Delta & (L \div)
\end{array}
$$

3. Admissibility of these rules follows by expansion of $\square$.

$(\mathrm{T})$

$$
\begin{aligned}
& \Gamma, A \vdash \Delta \\
& \Gamma \vdash-A, \Delta \\
& \Gamma, \div-A \vdash \Delta
\end{aligned}
$$
$\left(\mathrm{S} 4^{\prime}\right)$ $\div \Gamma \vdash A$

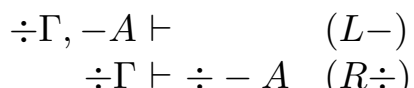

(S4) is just a special case of (S4').

Remark 5.4. Notice that, given the rule $\left(L^{\prime} \div\right)$, the rule $(L \div)$ becomes admissible, simply as its special case. However, the latter is invertible while the former is not. (Invertibility may fail whenever, semantically, $\div A \neq-A$. It obtains whenever this equality holds, e.g., when $A$ is closed, in particular, has the form $-\div A^{\prime}$.) Non-invertibility of $\left(L^{\prime} \div\right)$ is suggested by the mere fact that the proof of its admissibility uses $(W)$. (Analysis showing that such a use is required might even establish non-invertibility.) Since all rules of LIC, except for $(W)$, are invertible, the proofs identify explicitly the "non-invertible transitions" needed, typically, in the intuitionistic logic.

$(W)$ is necessary because of $(R \div)$. Given only the classical rules, $(W)$ can be made admissible by generalizing the form of the axioms to $\Gamma \vdash \Delta$ where $\Gamma \cap \Delta \neq \emptyset$. However, in the presence of $(R \div)$, this is no longer possible. Without $(W)$ no sequent of the form $\vdash \div A, \div B$ would be provable. This strengthenes the conjecture that no sound and complete set of invertible rules can be designed for the intuitionistic logic.

Note, furthermore, that the rule $(L \div)$ involves implict contraction, as the principal formula $\div A$ is retained in the premiss. ${ }^{4}$ Replacing this rule with $\left(L^{\prime} \div\right)$ would require us to view each side of the sequents as a multiset, and not a set, of formulae, and would demand explicit contraction rule. For instance, the bottom part of the proof from example 5.2, would have to be modified as follows:

\footnotetext{
${ }^{4}$ Following the standard terminology, e.g., [8], we call a formula appearing explicitly in the conclusion of a rule its "principal formula".
} 


\begin{tabular}{|c|c|}
\hline$\div(A \vee \div A)$ & $\vdash \quad \div A \vee A$ \\
\hline$\div(A \vee \div A), \div(A \vee \div A)$ & $\vdash$ \\
\hline$\div(A \vee \div A)$ & $\vdash$ \\
\hline
\end{tabular}

\subsection{Some auxiliary results}

The lemmata from this subsection are used in the completeness proof in 5.2.

LEMmA 5.5. Each of the follwing formulae is provable:

i. $\vdash A \rightarrow A$

ii. $\vdash(A \rightarrow B) \rightarrow((B \rightarrow C) \rightarrow(A \rightarrow C))$

iii. $\vdash A \rightarrow A \vee B$

iv. $\vdash(A \rightarrow C) \rightarrow((B \rightarrow C) \rightarrow(A \vee B \rightarrow C))$

v. $\vdash A \wedge B \rightarrow A$

vi. $\vdash(C \rightarrow A) \rightarrow((C \rightarrow B) \rightarrow(C \rightarrow(A \wedge B)))$

vii. all the above with $X \rightarrow Y$ replaced by $X \hookrightarrow Y$, i.e., by $\div-(X \rightarrow Y)$

viii. $\vdash(A \rightarrow(B \rightarrow C)) \leftrightarrow(A \wedge B \rightarrow C)$

ix. the above with $A$ repalced by $\div A$ and $\rightarrow$ by $\hookrightarrow$

x. $\vdash A \wedge-A \rightarrow B$

xi. $\vdash(A \rightarrow(A \wedge-A)) \rightarrow-A$

xii. the two above with,$- \rightarrow$ replaced by $\div, \hookrightarrow$

xiii. $\vdash A \vee-A$

Proof. $\mathrm{i}-\mathrm{-vi}$, viii, $\mathrm{x}$, xi and xiii follow since our calculus includes the classical sequent calculus. The respective "intuitionistic" versions, vii, ix, xii follow by appropriate removal of $\div-$ using $(S 4),(T)$. We give only one example terminating the proof once it arrives at a propositional form.

ix. $\rightarrow$ :

$$
\begin{aligned}
& \begin{array}{lcc}
A, B \vdash C, A \quad \frac{B \rightarrow C, A, B \vdash C}{\div-(B \rightarrow C), A, B \vdash C \quad(T)} \\
A \rightarrow \div-(B \rightarrow C), A, B \vdash C
\end{array}(L \rightarrow) \\
& A \rightarrow \div-(B \rightarrow C) \vdash A \wedge B \rightarrow C \\
& \div-(A \rightarrow \div-(B \rightarrow C)) \vdash A \wedge B \rightarrow C \\
& \div-(A \rightarrow \div-(B \rightarrow C)) \vdash \div-(A \wedge B \rightarrow C) \\
& \vdash \div-(A \rightarrow \div-(B \rightarrow C)) \rightarrow \div-(A \wedge B \rightarrow C) \\
& (R \rightarrow),(L \wedge) \\
& \vdash \div-(\div-(A \rightarrow \div-(B \rightarrow C)) \rightarrow \div-(A \wedge B \rightarrow C)) \\
& \vdash(A \hookrightarrow(B \hookrightarrow C)) \hookrightarrow(A \wedge B \hookrightarrow C)
\end{aligned}
$$




$$
\begin{aligned}
\leftarrow: & \div A \wedge B \rightarrow C, \div A \vdash B \rightarrow C \\
& \div-(\div A \wedge B \rightarrow C), \div A \vdash B \rightarrow C \\
& \div-(\div A \wedge B \rightarrow C), \div A \vdash \div-(B \rightarrow C) \\
& \div-(\div A \wedge B \rightarrow C) \vdash \div A \rightarrow \div-(B \rightarrow C) \\
& \div-(\div A \wedge B \rightarrow C) \vdash \div-(\div A \rightarrow \div-(B \rightarrow C)) \\
& \vdash-(\div A \wedge B \rightarrow C) \rightarrow \div-(\div A \rightarrow-(B \rightarrow C)) \\
& \vdash \div-(\div-(\div A \wedge B \rightarrow C) \rightarrow \div-(\div A \rightarrow \div-(B \rightarrow C)))
\end{aligned}
$$

The statements $\mathrm{i}$-vi, together with $\mathrm{x}$, xi and xiii imply that the Lindebaum algebra for LIC, $\mathbf{L}$, is boolean and we will use this in the proof of completeness below. The statements vii, ix and xii, apply to all elements except for the one direction of ix, where the restriction to open elements, $\div A$, is needed. As these statements apply, in particular, to all open elements, this means that the Lindenbaum algebra actually contains a Heyting algebra of open elements with $\hookrightarrow$ being the relative pseudo-complement and $\div$ pseudo-complement. This fact will not enter directly into the completeness proof, but it is related to the following lemma, which will ensure that $\mathbf{L}$ is actually an $\mathcal{I C}$-algebra.

Lemma 5.6. The following are provable:

$$
\begin{aligned}
& \text { i. } \div A \vdash-A \\
& \text { ii. } \div A \vdash \div-\div A \text { and } \div-\div A \vdash \div A \\
& \text { iii. } \div(A \vee B) \vdash \div A \wedge \div B \text { and } \div A \wedge \div B \vdash \div(A \vee B) \\
& \text { iv. } \vdash \div(A \wedge-A)
\end{aligned}
$$

Lemma 5.7. The following rules are admissible:

$$
\begin{aligned}
\text { i. } & \frac{\vdash A \rightarrow B}{\vdash \div B \rightarrow \div A} \\
\text { ii. } & \frac{\Gamma \vdash \bigvee \Delta, \Delta^{\prime}}{\Gamma \vdash \Delta, \Delta^{\prime}} \text { and } \frac{\bigwedge \Gamma, \Gamma^{\prime} \vdash \Delta}{\Gamma, \Gamma^{\prime} \vdash \Delta}
\end{aligned}
$$

Proof. i. The last step in the proof of $\vdash A \rightarrow B$ must apply $(R \rightarrow)$ to $A \vdash B$, so we get

$$
\begin{array}{ll}
A \vdash B & \\
\div B, A \vdash & \left(L^{\prime} \div\right) \\
\div B \vdash \div A & (R \div)
\end{array}
$$

ii. Consider the first of the rules. We proceed by induction on the number $n$ of disjuncts in $\bigvee \Delta$. The basis $n=1$ is obvious, so assume IH for $\bigvee \Delta$ and a proof of $\Gamma \vdash \vee \Delta \vee D, \Delta^{\prime}$. Consider the first place $l$. in the bottom-up 
proof (i.e., the lowest place when viewed top-down) where this disjunction is the principal formula. It may be introduced by $(R \vee)$ or by $(\mathrm{W})$. In the first case we have the following situation:

$$
\begin{aligned}
& l-1 . \quad \Gamma^{\prime \prime} \vdash \bigvee \Delta, D, \Delta^{\prime \prime} \\
& \text { l. } \quad \Gamma^{\prime \prime} \vdash \vee \Delta \vee D, \Delta^{\prime \prime} \quad R \vee \\
& \vdots \quad \vdots \\
& \text { z. } \quad \Gamma \vdash \bigvee \Delta \vee D, \Delta^{\prime}
\end{aligned}
$$

By IH, we have a proof $l^{\prime}$. $\Gamma^{\prime \prime} \vdash \Delta, D, \Delta^{\prime \prime}$. (The situation is entirely analogous if $\bigvee \Delta \vee D$ is split in any other way as $\bigvee \Delta_{1}, \bigvee \Delta_{2}$.) Since the disjunction is not processed between $l$ and $z$, the rule $(R \div)$ could not be applied anywhere between $l$ and $z$. But then, since all the other rules are context insensitive, we can reuse the derivation $l \ldots z$ starting from $l^{\prime}$ instead. This will yield a proof $z^{\prime} . \Gamma \vdash, \Delta, D, \Delta^{\prime}$.

If $\bigvee \Delta \vee D$ is introduced at $l$ by (W), we simply introduce $\Delta, D$ instead and copy the rest of the derivation which is possible by the same argument as above.

The proof of the other rule proceeds analogously by induction on the number $n$ of conjuncts in $\bigwedge \Gamma$, with the trivial basis case $n=1$.

Note that the empty lhs in i. is essential - the rule $\frac{\Gamma \vdash A \rightarrow B}{\Gamma \vdash \div B \rightarrow \div A}$ is

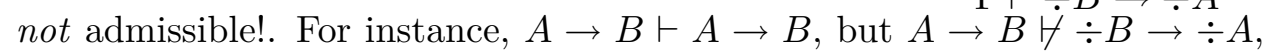
which would be unsound. E.g., $-A \cup B \nsubseteq-\div B \cup \div A$, if we take $-\div B=$ $B \subset \div A \subset-A=-(\div-A)$.

\subsection{The completeness proof}

5.2.1. The construction of the Lindenbaum algebra $\mathbf{L}$ for $L I C$, over a given alphabet $X$, follows [7] (numbers in square parantheses refer to the results given there). Let $\mathcal{F}(X)$ denote the set of all formulae over the alphabet $X$ where, for convenience, we use the symbols $\cup, \cap$ instead of $\vee, \wedge$. We define:

1. $\forall A, B \in \mathcal{F}(X): A \leq B \Longleftrightarrow \vdash A \rightarrow B$

2. $\forall A, B \in \mathcal{F}(X): A \simeq B \Longleftrightarrow A \leq B$ and $B \leq A$.

3. the domain of $\mathbf{L}$ is $\mathcal{F}(X) / \simeq$, and for op $\in\{-, \div, \cup, \cap, \rightarrow\}: o p^{\mathbf{L}}\left(\left[A_{i}\right]\right)=$ $\left[o p\left(A_{i}\right)\right]$

5.2.2. 5.5.i-ii ensure that $\leq$ is a quasi-ordering over $\mathcal{F}(X)$ and hence $\simeq$ is 
an equivalence. It induces an ordering (reflexive, transitive, antisymmetric) over $\mathcal{F}(X) / \simeq$ with $[A] \subseteq[B] \Leftrightarrow A \leq B$. Thus $[A] \subseteq[B] \Leftrightarrow \vdash A \rightarrow B$.

Now, 5.5.iii-vi (iii and $\mathrm{v}$ apply to both arguments, only one of which was mentioned) ensure that $\mathbf{L}$ is a lattice and $\simeq$ a congruence wrt. $\cup, \cap$ [VI.10.3]. When also viii, $\mathrm{x}, \mathrm{xi}$, xiii of 5.5 hold, $\mathbf{L}$ is a boolean algebra and $\simeq$ is a congruence also wrt. to - , [VI.10.6].

5.2.3. That $\simeq$ is a congruence also wrt. $\div$ follows by Lemma 5.7.i which implies that if $A \leq B$ and $B \leq A$, then also $\div B \leq \div A$ and $\div A \leq \div B$. So $\mathbf{L}$ is well-defined.

5.2.4. Thus $\mathbf{L}$ is a boolean algebra, and we verify that it is also $\mathcal{I C}$, i.e.,

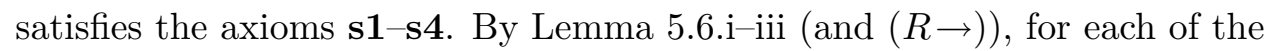
axioms s1-s3, $l=r$, the respective implications $\vdash l \rightarrow r$ and $\vdash r \rightarrow l$ are provable. Hence $\mathbf{L}$ satisfies these axioms. By 5.5.x, $\mathbf{0}=[A \wedge-A]$, and by 5.6.iv we have that $\mathbf{L} \models \div \mathbf{0}=\mathbf{1}$, i.e., also $\mathbf{s} \mathbf{4}$ holds in $\mathbf{L}$.

5.2.5. We consider only the canonical valuation of formulae in $\mathbf{L}$, i.e., one given by $c(p)=[p]$ for $p \in X$, which extends to $c(A)=[A]$ for all formulae $A$. $\mathbf{L} \models_{c} A$ means thus that $[A]=\mathbf{1}$ under the canonical valuation, where $\mathbf{1}=[A \vee-A]=[A \rightarrow A]$.

5.2.5.i. $\vdash A \Leftrightarrow \mathbf{L} \models_{c} A$, [VI.10.4]. If $\vdash A$, then also (by $(L W)$ and $(R \rightarrow)$ ) $\vdash(A \rightarrow A) \rightarrow A$, so $\mathbf{1}=[A \rightarrow A] \subseteq[A]$. Conversely, if $[A]=\mathbf{1}$, then $[A \rightarrow A] \subseteq[A]$ and by $5.2 .2 \vdash(A \rightarrow A) \rightarrow A$. Hence also $A \rightarrow A \vdash A$. Since $\vdash A \rightarrow A$ by 5.5 .i so, by admissibility of (cut), we conclude $\vdash A$.

5.2.5.ii. LIC is consistent (does not prove some formula) iff $\mathbf{L}$ is not degenerate, i.e., contains at least two elements, [VI.10.7]. For by 5.2.5.i $\forall$ $A \Leftrightarrow[A] \neq \mathbf{1}$, which means that the domain of $\mathbf{L}$ has at least two distinct elements.

( $p \rightarrow-p$, for $p \in X$, gives an example of an unprovable $A$. I.e., $\mathbf{L}$ is non-degenerate.)

5.2.6. If for some formula $\forall A$ then, by 5.2.5.i, $[A] \neq \mathbf{1}$, i.e., $\mathbf{L} \not \nvdash_{c} A$. That is, $\mathbf{L} \not \models A$ and, since $\mathbf{L} \in \mathcal{I C}$ by $5.2 .4, \mathcal{I C} \not \models A$.

Thus, combined with Lemma 5.1, we have for any $A: \vdash A \Leftrightarrow \models A$.

5.2.7. The general statement follows: $\Gamma \models \Delta \Leftrightarrow \cap \Gamma \subseteq \cup \Delta \Leftrightarrow-\cap \Gamma \cup$ $\cup \Delta=1$, which by the above obtains iff $\vdash \wedge \Gamma \rightarrow \bigvee \Delta$. But any proof of the latter must begin (bottom-up) with $\wedge \Gamma \vdash \bigvee \Delta$. Lemma 5.7.ii gives then $\Gamma \vdash \Delta$. 


\subsection{Decidability}

A tree of all possible (and attempted) derivations of a given sequent $S$ is constructed bottom-up starting with $S$ in the root node. From each node, we split the tree into $n$ branches where $n$ is the number of all possible applications of all rules to the sequents contained in the current node. The subsequent node in each branch contains all the premisses of the respective rules' application. When no rule is applicable to the (set of sequents in a) node, the branch terminates. It terminates with success when the final node contains only instances of axioms, and with failure otherwise.

All rules have the subformula property. Applied bottom-up they also reduce the complexity of the sequent (measured by the number of connectives), with the only exception of $(L \div)$ which preserves the principal formula in the premiss. Hence, branches whose nodes contain sequents with $\div A$ on the left of $\vdash$, may be infinite. Then such branches contain also infinitely many applications of $(L \div)$. But due to the subformula property, in any such branch there will be (infinitely many) nodes with identical (sets of) sequents. We terminate a branch once such a repetition occurs.

Hence all branches terminate and the tree gives a proof iff at least one branch terminates with success. Putting the possible worries about the branching and complexity aside, we see that LIC is decidable. Recalling the grammars $\beta, \iota, \mu$ from page 197 , we thus obtain in one stroke decidability of classical, intuitionistic and S4 logics.

\section{A concluding note on a possible reading}

One can attempt a variety of readings of the operation $\div$ and, consequently, of the modalities $\div-$ and $-\div$. To avoid torturous arguments, let us follow Heyting's contention that while the classical logic addresses the ontological aspect of the world, the intuitionistic logic addresses the (finite/constructive) epistemic aspect. Accepting this general statement, the Heyting substructure of an $\mathcal{I C}$-algebra can be seen as a subuniverse of epistemic approximations to objects which, in general, may lay outisde it in the classical universe. All elements of this universe can be seen as objects knowledge might be about, while the opens as the objects knowledge is actually using. While IL is only logic of the solipsistic knowledge unrelated to any world outside its finite constructions, the modal logics arise from the interacion between these two dimensions. 
6.1. The reading of the arising modalities will depend on the more specific reading of the opens. Given the above view of opens as the epistemic elements, and the fact that $\div x$ is open, one might propose to read it as some form of epistemic impossibility. ${ }^{5}$

$\square x=\div-x$ becomes then the (epistemic) impossibility of the negation of $x$. Thus, $\square$ read as knowledge, becomes an epistemic impossibility (unimaginability) of the contrary. But this is no different from necessity, at least if we grant its epistemic character. At least to the common-sense, necessity is simply impossibility of accepting other alternatives, as when we say: "This is unavoidable!" Surely, few things are ever unavoidable/necessary in the strict sense of logical impossibility. In the more mundane situations, logical impossibility is replaced by milder, that is, more epistemic predicates: irrelevancy, implausibility or incapacity, and $x$ appears unavoidable just when its contrary falls under some such predicate.

Possibility, having classical negation as the main operator, acquires a more ontological character. $\diamond x=-\div x \supseteq \div \div x$, i.e., $x$ is possible not only when its impossibility appears (is epistemically) impossible, $\div \div x$, but also when it actually - ontologically - does not obtain, $-\div x$.

6.2. The $\mathcal{I C}$-formulation does not commit one to any specific choice as to where the line separating the ontological from the epistemic should be drawn. It only acknowledges the distinction between the two, and obtains modalities out of their combination. Necessity acquires an epistemic aspect and knowledge turns out as ... its synonym - of course, knowledge understood not merely as an acceptance of a fact, but as inadmissibility of a contrary, we might say, as a justified belief.

6.3. The epistemic-ontological complementarity can be also read into $\mathrm{S} 5$ as presented here. We have seen that the s5 axiom amounts to equating the epistemic and the ontological negation when applied to the epistemic elements. The epistemic subuniverse can still be distinct from the ontological one, but it is itself a classical universe. This is the counterpart of the specific property of S5, namely, that any chain of modalities is equivalent ("collapses") to the rightmost one. In $\mathcal{I C}$-formulation, having once entered

\footnotetext{
${ }^{5}$ Speaking stricly and intuitionistically, such an impossibility amounts to the presence of a counter-proof, namely, of the proof $\div \Gamma, A \vdash \varnothing$, from which $\div \Gamma \vdash \div A$ follows by $(R \div)$. Observe that this rule implies that only other "epistemic" elements, $\div \Gamma$, can contribute to establishing the "epistemic" impossibility $\div A$ of $A$. Alternative formulations will appear with different variants of modal logics.
} 
the epistemic subuniverse (by means of $\div$ ), the $\div$ becomes,$- \div \div=-\div x$, and so no more properly modal operations are available. This seems to express well the traditional reading of S5 as the logic of metaphysical necessity: it comprises a subset of the actual universe (all necessary truths) which is itself governed by the same (classical) laws without any extraneous epistemic disturbances.

6.4. Working with the "possible world" semantics (of modal logic), one has been in need to repeatedly emphasize that "possible worlds" are not any strange other-worldly entities but simply possible variations of the states of affairs obtaining in the world we are actually living in. Some interpreters could take the phrase "possible worlds" more literally. Such an interpretative misuse is, however, grounded in the semantic formalism itself where, indeed, different possible worlds can have nothing ontological in common. If one points at one world claiming that this is the actual one, there is still nothing in the framework ensuring that all agents actually share in this particular world; there may even be agents to whom this world remains inaccessible. (A residual trace of the "common world" can be found, e.g., in the concept of rigid designators whose role (apart from giving an interpretation of proper names) is exactly to establish a common ontology shared by all possible worlds.)

The presented view resolves this problem by means of the distinction between the classical world of ontology and its intuitionistic substructure of epistemic approximations. A variety of possibilities is then simply a potential multiplicity of such "epistemic subuniverses" which all are substructures of the same (classical) world. Formally, one would simply introduce a multiplicity of $\div i$, one for each agent $i .^{6}$

6.5. To give an impression of such a modelling of multiple agents, let us give two simple examples for, respectively, S4 and S5.

6.5.i. Consider a simple classical world $\mathbf{B}=\mathcal{P}(\{a, b, c\})$ and its two epistemic substructures, Heyting algebras H1, H2. (We denote joins by con-

\footnotetext{
${ }^{6}$ Thus, the "philosophical" view of modalities might seem contrary to that involved in Kripke semantics. At the technical level, however, there is no need to posit any opposition since reachability relation can be recovered from the topological (and hence $\mathcal{I C}$ ) algebra applying Jónsson-Tarski's representation theorem (3.10 from [3], also chap. 5 of [1]).
} 
catenation, e.g., $a \cup b$ is written $a b$.)
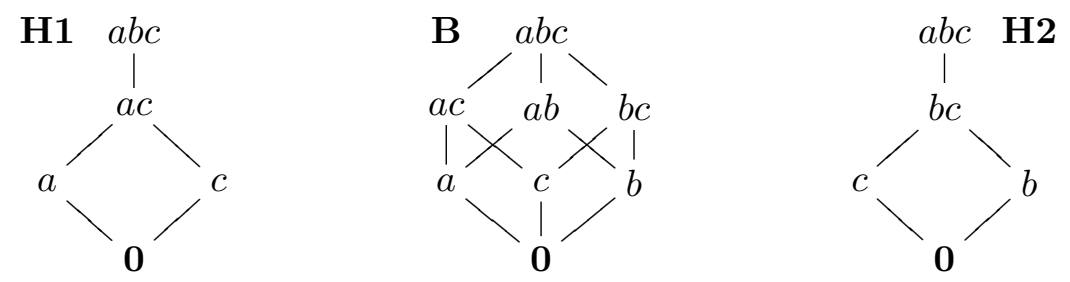

We have, for instance:

\begin{tabular}{rrr|c} 
& & H1 & H2 \\
1. & $-\quad c=$ & $a b$ & $a b$ \\
2. & $\div c=$ & $a$ & $b$ \\
3. & $\div-c=$ & $c$ & $c$ \\
4. & $-\div c=$ & $b c$ & $a c$ \\
5. & $\div \div c=$ & $c$ & $c$
\end{tabular}

\begin{tabular}{r|c|c} 
& H1 & H2 \\
\hline$-b=$ & $a c$ & $a c$ \\
$\div b=$ & $a c$ & $c$ \\
$\div-b=$ & $\mathbf{0}$ & $b$ \\
$-\div b=$ & $b$ & $a b$ \\
$\div \div b=$ & $\mathbf{0}$ & $b$
\end{tabular}

\begin{tabular}{r|c|c} 
& H1 & H2 \\
\hline$-b c=$ & $a$ & $a$ \\
$\div b c=$ & $a$ & $\mathbf{0}$ \\
$\div-b c=$ & $c$ & $b c$ \\
$-\div b c=$ & $b c$ & $a b c$ \\
$\div \div b c=$ & $c$ & $a b c$
\end{tabular}

The first table concerns the element $c$ present in both $\mathbf{H 1}$ and H2. The differences in rows 2 . and 4 . reflect the differences between the respective epistemologies. Reading $\div c$ as "recognized impossibility of $c$ ", for $\mathbf{H 1}$ it can be only $a$ while for $\mathbf{H 2}$ only $b$. This is then reflected in what appears as $c$ 's possibility in row 4 . In either case it can be $c$ itself, but for $\mathbf{H 1}$, possibly also $b$ - as it does not belong to its epistemic world, the possibilities it harbours are not recognizable by $\mathbf{H} \mathbf{1}$.

The two last tables concern elements which are in the epistemic world $\mathbf{H} 2$ but not $\mathbf{H 1}$. Thus, either $a$ or $c$ of $\mathbf{H 1}$ amount to impossibility of $b$, while for $\mathbf{H 2}$, it is only $c$. Dually, the necessity of $b$, row 3., does not obtain in $\mathbf{H 1}$, while it is present in $\mathbf{H 2}$ as the element $b$ itself. (In the third table, although $b c \notin \mathbf{H} \mathbf{1}$, its necessity still obtains as the element $b-$ the best available approximation of this epistemically absent element.) The possibility of $b$, row 4 ., is not however absent for $\mathbf{H 1}$, although it does not meet any elements in $\mathbf{H 1}$ - it is an "external" possibility, obtaining only due to the ontological structure of the whole B. For H2, this possibility is further extended by the element $a$ which is not part of his epistemic world (and hence might, potentially for $\mathbf{H 2}$, harbour the possibility of $b$, even if it actually does not).

6.5.ii [S5] Consider the same $\mathbf{B}$ as above and two S5 epistemic substructures H1, H2. Since s5 axiom makes complements of opens open, the 
Heyting substructure becomes a boolean algebra where all opens are also closed.
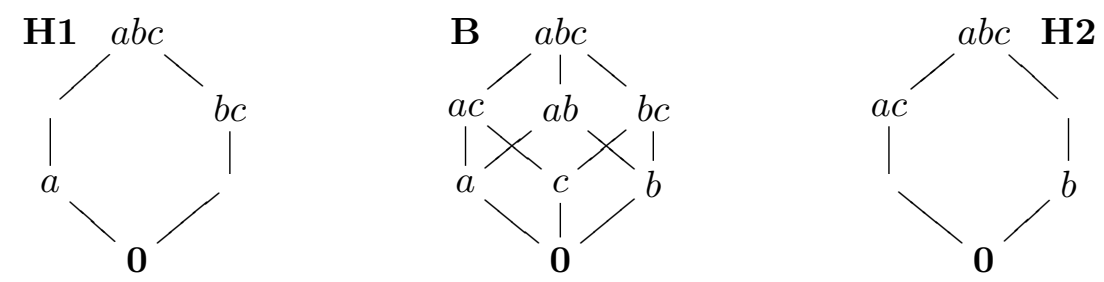

We have, for instance:

\begin{tabular}{rrr|c} 
& & H1 & H2 \\
1. & $-c=$ & $a b$ & $a b$ \\
2. & $\div c=$ & $a$ & $b$ \\
3. & $\div-c=$ & $\mathbf{0}$ & $\mathbf{0}$ \\
4. & $-\div c=$ & $b c$ & $a c$ \\
5. & $\div \div c=$ & $b c$ & $a c$
\end{tabular}

\begin{tabular}{r|c|c} 
& H1 & H2 \\
\hline$-b=$ & $a c$ & $a c$ \\
$\div b=$ & $a$ & $a c$ \\
$\div-b=$ & $\mathbf{0}$ & $b$ \\
$-\div b=$ & $b$ & $a b$ \\
$\div \div b=$ & $b$ & $a b$
\end{tabular}

\begin{tabular}{r|c|c} 
& H1 & H2 \\
\hline$-b c=$ & $a$ & $a$ \\
$\div b c=$ & $a$ & $\mathbf{0}$ \\
$\div-b c=$ & $b c$ & $b$ \\
$-\div b c=$ & $b c$ & $a b c$ \\
$\div \div b c=$ & $b c$ & $a b c$
\end{tabular}

Note that although the epistemic substructures are now boolean algebras, the epistemic negation $\div$ does not coincide with the ontological one - . The difference concerns the epistemically absent elements. Thus, for instance, in the first table, $c$ is epistemically absent from H1, but its impossibility, $\div c$, amounts only to the epistemically available contraries, namely, $a$, and not to its ontological negation $a b$. Likewise, in the third table, $b c \notin \mathbf{H 2}$, but its epistemic impossibility amounts to contraditiction $\mathbf{0}$, although ontologically it can be also obtained as $a$.

6.6. Finally, let us observe an entirely different aspect of epistemic modelling. The view of knowledge as the ability to draw and relate distinctions indicates intimate relations to topology. The fact that topological opens form a Heyting algebra provided the basis, in [9] (and in the tradition of formal topology), for viewing them as representing finite observations allowing to draw distinctions. Such a view of knowledge emerges naturally from the topological interpretation of $\mathcal{I C}$-algebras.

Consider, for instance, a classical world $\mathcal{P}(\{a, b, c, d\})$ and a Heyting substructure containing (the opens) $\{\emptyset, a, b, a b, a b c d\}$ with $\emptyset=\mathbf{0}$ and $a b c d=\mathbf{1}$. Following the topological view, this amounts to (being capable of) distinguishing $a$ from $b$ (having disjoint opens covering each of them). However, $c$ and $d$ fall outisde the epistemic world and, consequently, they (or their join) 
are indistinguishable by the available epistemic means (table on the left). Also, no interaction of these elements with the available $a, b$ will uncover any difference between them (table on the right):

\begin{tabular}{r|c|c|c} 
& $c$ & $d$ & $c d$ \\
\hline- & $a b d$ & $a b c$ & $a b$ \\
$\div$ & $a b$ & $a b$ & $a b$ \\
$\div-$ & $\mathbf{0}$ & $\mathbf{0}$ & $\mathbf{0}$ \\
$-\div$ & $c d$ & $c d$ & $c d$
\end{tabular}

\begin{tabular}{c|c|c|c} 
& $a c$ & $a d$ & $a c d$ \\
\hline- & $b d$ & $b c$ & $b$ \\
$\div$ & $b$ & $b$ & $b$ \\
$\div-$ & $a$ & $a$ & $a$ \\
$-\div$ & $a c d$ & $a c d$ & $a c d$
\end{tabular}

Expanding the epistemic base with, say, recognition of the element $c$ will, of course, lead to new distinctions, e.g., $\div c=a b \neq a b c=\div d$.

Viewing the epistemic elements as the distinctions one is capable of recognizing, the modalities arise now from the interaction between such distinctions and the ontological ones which, however, remain epistemically indistinct. One might even be tempted to read now $\div x$ as the impossibility to apprehend/recognize $x$, with the consequences for:

- 'necessity' of $x=\div-x=$ impossibility to apprehend the negation of $x$ and

- 'possibility' of $x=-\div x=$ the absence of impossibility of apprehension of $x$.

Acknowledgments. Paul Simon Svanberg deserves thanks for interesting discussions and suggestions related to the contents of this paper.

The work has been partially supported by the Norwegian Research Council, under the project MoSIS.

\section{References}

[1] P. Blackburn, M. de Rijke, and Yde Venema, Modal Logic. Cambridge University Press, 2001.

[2] A. Horn, "Free s5 algebras", NotreDame Journal of Formal Logic 29(1) (1978), 189-191.

[3] B. Jónsson and A. Tarski, "Boolean algebras with operators I", American J. Mathematics 73 (1951), 891-939.

[4] H.M. MacNeille, "Partially ordered sets", Transactions of the American Mathematical Society 42 (1937), 416-460. 
[5] J.C.C. McKinsey and A. Tarski, "The algebra of topology", The Annals of Mathematics 45(1) (1944), 141-191.

[6] J.C.C. McKinsey and A. Tarski, "On closed elements in closure algebras", The Annals of Mathematics 47(1) (1946), 126-162.

[7] H. Rasiowa and R. Sikorski, The Mathematics of Metamathematics. PWN, Warszawa, 1963.

[8] A.S. Troelstra and H. Schwichtenberg, Basic Proof Theory. Cambridge University Press, 2 edition, 2000.

[9] S. Vickers, Topology via Logic. Cambridge University Press, 1989.

[10] M. Walicki, "Modalities as interactions between the classical and the intuitionistic logics", Technical Report 330, Department of Informatics, University of Bergen, 2006.

Michą WALiCKI

Department of Informatics

University of Bergen

Norway

michal@ii.uib.no 